\title{
REFLEXIONES DESDE LA REALIDAD CHILENA ACERCA DE LAS OPORTUNIDADES Y DESAFÍOS DE LA EDUCACIÓN UNIVERSITARIA EN EL SIGLO XXI1
}

\author{
Jaime Caiceo Escudero2
}

\section{RESUMEN:}

Al terminar la primera década del nuevo siglo es importante hacer un alto y reflexionar acerca de las oportunidades y desafíos de la universidad para las décadas restantes. En este sentido, se reflexiona a partir de los principios fundacionales de la misma en la Edad Media; ello conduce a que esos principios fueron exigir respeto a su autonomía, a abarcar todo el saber humano, a que los maestros y estudiantes actuaran en forma unida y colaborativa, a que sólo es patrimonio de ella la entrega de todos los grados académicos (bachiller, licenciado, magíster y doctor),, a que haya mayor debate o discusión en el ejercicio de la investigación y la docencia universitaria y que exista el derecho a la huelga para lograr los puntos anteriores. A partir de esos principios se analizan los problemas actuales de la universidad, especialmente en América Latina y Chile: Universidad para todos, la inclusión, la acreditación y el intercambio de maestros y estudiantes.

Palabras Claves: Educación Universitaria/ Origen de la Universidad/ Proyecciones de la Universidad para el siglo XXI/ Universidad Latinoamericana/ Universidad Chilena.

\section{REFLECTIONS FROM REALITY ABOUT CHILEAN OPPORTUNITIES AND CHALLENGES OF HIGHER EDUCATION IN THE XXI CENTURY}

\begin{abstract}
:
After the first decade of the new century, it is important to pause and reflect on the opportunities and challenges provided by universities for the remaining decades. In this sense, a reflection on the founding principles in the Middle Ages leads to principles of demanding respect for their autonomy, covering all human knowledge, promoting collaborative work between teachers and students which is considered as heritage of all academic degrees (bachelor, licensed, master and doctor), having more opportunities for debates and discussion in the field of research and university teaching and having the right to come out on strike to achieve these points mentioned above. From these principles we analyze the current problems of universities, especially in Latin America and Chile: University for all, including accreditation and exchange of teachers and students.

Keywords: Higher education / Origin of University/ University projections for the XXI Century /Latin American University/ Chilean University.
\end{abstract}

\section{I.- Introducción}

Producto de la globalización existente en el mundo desde hace dos décadas, se han comenzado a producir una serie de cambios en la sociedad y en la educación; las universidades no están exentas de ello; por lo mismo, el proceso de internacionalización de las mismas es una realidad que se ha acrecentado en los últimos años a nivel mundial y, por cierto, también en Chile. 
El objeto de este trabajo es reflexionar acerca de las oportunidades y desafíos de la educación universitaria para el nuevo siglo, teniendo como trasfondo la realidad internacional, pero especialmente la chilena ${ }^{3}$.

\section{II.- La universidad y su origen}

Al iniciar cualquier reflexión es imprescindible remontarse al concepto original y cómo surgió el objeto de estudio; en este caso, la universidad. Justamente, por el mundo pragmático en que vive la sociedad actual, se hace más necesario tal procedimiento metodológico.

La palabra universidad deriva del latín, universitas, que significa corporación o gremio y en este sentido hacía referencia al gremio de maestros y estudiantes. Por lo tanto, nos encontramos con que la universidad surgió en la Alta Edad Media, en torno al siglo XII, en el contexto de las corporaciones que fueron formándose en las ciudades que practicaban un mismo oficio para defender sus intereses; de esta forma, los artesanos del espíritu, es decir, los intelectuales, formaron su propia corporación: la Universidad. De esta forma, las universidades van surgiendo como una corporación más. Es la corporación o gremio o universidad de maestros y estudiantes, es decir, de todos los intelectuales que viven en la misma ciudad. Tanto gremios como universidades o comunas les toca ahora luchar por conquistar su autonomía contra los poderes eclesiásticos y laicos y contra otras corporaciones y comunas. Esta autonomía se alcanza gracias a la cohesión y decisión de sus miembros y a la presión ejercida por la amenaza y empleo efectivo de la huelga y la secesión ${ }^{4}$.

Fue así, por lo tanto, que se obtuvo (1) la autonomía jurisdiccional, (2) el derecho a huelga y secesión y (3) el monopolio de los grados académicos. La Iglesia Católica jugó un rol vital en estos logros universitarios, entregando autonomía, privilegios, estatutos, apoyo económico y reconocimiento a la labor intelectual, pues toma conciencia que ésa es una forma muy adecuada de estudiar más a fondo la fe, preservarla y difundirla. Para estudiar el saber de la época, la universidad se agrupó en cuatro facultades. Teología, arte, derecho y medicina, otorgando cuatro grados académicos: bachiller, licenciado, magister y doctor. Los métodos de enseñanza en la universidad medieval se pueden reducir a tres, a saber: La Lectio o lección, la quaestio o cuestión y la disputatio o disputa. Este último método es la expresión del culmen de la pedagogía medieval, pues es una pedagogía activa que exigía que estudiantes y profesores estuvieran al tanto de los problemas cotidianos (nacionales, internacionales y regionales), manteniendo así a la universidad en contacto permanente con la vida.

La primera universidad conocida es la de Bolonia en Italia hacia fines del siglo XI (1088 aproximadamente), centrada especialmente en el estudio del derecho romano por el redescubrimiento del Código de Derecho Civil de Justiniano. Luego vendrán la Universidad de París, en Francia, reconocida en 1200, la de Oxford (1208) y Cambridge (1209) en Inglaterra 5 .

Durante la colonia se fundaron en el entonces Reino de Chile dos universidades católicas, dependientes de la Orden de Santo Domingo -Universidad Santo Tomás- y de la Compañía de Jesús -Universidad de San Miguel-, respectivamente, comenzando a operar ambas en 1622 y una universidad dependiente de la corona -la Real Universidad de San Felipe- en 1747. 
Con el comienzo de la República en Chile se fundaron en Santiago la Universidad de Chile en 1842 -universidad pública y de inspiración laica- y la Universidad Católica de Chile en 1888 -universidad particular dependiente de la Iglesia Católica y como reacción al laicismo imperante en la universidad pública-; durante el siglo XX y hasta antes de la dictadura militar se fundaron 6 universidades más, tanto en Santiago como en Provincias: La Universidad de Concepción -universidad particular dependiente de la masonería, instalada en la ciudad de Concepción- en 1920; la Universidad Técnica Federico Santa María -universidad particular laica, instalada en la ciudad de Valparaíso- en 1926; la Universidad Católica de Valparaíso -universidad particular dependiente de la Iglesia Católica, instalada en la ciudad de Valparaíso - en 1928; la Universidad Técnica del Estado -universidad pública originada a partir de la Escuela de Artes y Oficios fundada en 1849 en Santiago- en 1947, la Universidad Austral de Chile -universidad particular laica, instalada en la ciudad de Valdivia- en 1954 y la Universidad Católica del Norte -universidad particular dependiente de la Iglesia Católica, radicada en la ciudad de Antofagasta- en $1964^{6}$.

\section{III.- Desafíos y oportunidades de las universidades}

A partir de lo expuesto en el punto anterior, se puede precisar que el gran desafío que tienen las universidades hacia el futuro está en:

1. Mantener y profundizar su autonomía con el objeto de logar cumplir cada vez mejor el desarrollo de las artes, de la ciencia, de la filosofía y de la teología, a través de la investigación interdisciplinaria, formando grupos de investigadores con nexos con otros grupos de investigadores no sólo de universidades nacionales sino que ojala internacionales e intercontinentales. El diálogo interdisciplinario cada vez es más urgente, pues las ciencias están entrelazadas entre sí, pero también lo están con las artes, la filosofía y la teología. No basta sólo el diálogo ciencia fe que promueve la Iglesia Católica para sus universidades confesionales sino que con los descubrimientos tan profundos, radicales y desconcertantes que realizan hoy los científicos, la presencia de la ética es cada vez más una necesidad; la ciencia por sí misma no es buena ni mala, pero puede producir mucho daño (la energía atómica con fines bélicos) o mucho bien (la energía atómica para las radiaciones cancerígenas); por lo mismo, los científicos necesitan de la iluminación de la ética para encausar debidamente sus descubrimientos.

2. Deben abarcar todo el saber humano en diferentes facultades, llegando a formar lo que se denomina una universidad compleja. Resulta paradojal que se hayan fundado entidades con el nombre de universidad y el saber lo restrinjan sólo a un aspecto, ingeniería, educación, derecho. El gran desafío para cumplir adecuadamente lo insinuado en el punto anterior es tener universidades complejas, es decir, entidades en las cuales estén presente las artes, las diferentes ciencias humanas y ciencias naturales, las matemáticas, la filosofía y la teología. En Chile, por ejemplo, cuando no existían universidades católicas durante gran parte del siglo XIX, la Universidad de Chile -entidad pública fundada en 1842- tenía entre una de sus facultades, la de Teología. Las facultades, a su vez, se deben dividir en departamentos, siendo éstos, la célula básica de una universidad, en los cuales se den los grupos de trabajo que investigan, entregan docencia actualizada y difunden el saber a través de actividades de extensión, congresos y publicaciones. 
3. Los maestros de una misma universidad o de diferentes universidades deben estar unidos, agrupados e interrelacionados, aprovechando las redes actuales y futuras de conexión: De esta manera se logra mejor el descubrimiento de tantas realidades del universo, del mundo y del propio ser humano que aún no se nos han develado; el científico no inventa las leyes de la naturaleza, sólo las descubre; un pequeño grano de arena descubierto aquí con otro descubierto allá pueden ayudarnos a explicar una nueva realidad.

4. La huelga y la secesión son armas que no hay que descuidar ${ }^{7}$, especialmente cuando algunas universidades deben servir a sus dueños (empresarios del dinero) y no a su razón de ser, el saber: Cuando los países en la actualidad han ido disminuyendo los presupuestos universitarios se tiene la tentación de sacrificar el sentido mismo de la universidad, sirviendo a grupos económicos determinados que ofrecen entregar financiamiento; por lo mismo, no hay que caer en la tentación fácil. Distinto es, por ejemplo, lo que sucede en Chile, gracias a la ley de donaciones: una empresa aporta recursos financieros a una universidad y lo descuenta de sus impuestos; la universidad emplea esos recursos en sus propios proyectos. Por lo tanto, para cumplir adecuadamente el rol universitario no hay que olvidar la autonomía y ello, incluye la autonomía presupuestaria que los países deben entregar directa o indirectamente; de lo contrario, habrá que recurrir a las armas históricas.

5. Defender el otorgamiento de los grados académicos cómo algo exclusivo de ellas. Suena insólito, pero entidades no universitarias desean entregar los grados académicos que son propio de aquéllas. Para evitar esta situación en Chile se dictaron una serie de leyes que fundamentan la educación superior, dejando explícitamente indicado que sólo las universidades otorgan los grados académicos ${ }^{8}$ y que los institutos profesionales ${ }^{9}$ o centros de formación técnica ${ }^{10}$ sólo pueden otorgar títulos profesionales o técnicos, según corresponda.

6. Recuperar el método de la disputa: Ello implica que tanto los maestros como los estudiantes deben estar al día en lo que sucede en el mundo (acontecimientos) y en el desarrollo de las artes, las ciencias, la filosofía y la teología, a fin de poder discutir el saber de las diferentes ciencias y disciplinas y tomar conciencia hacia dónde va la humanidad. No hay que olvidar que la ciencia está al servicio del hombre y no al revés. Tampoco hay que olvidar que con la discusión se avanza al poder defender cada persona con fundamentos en la realidad y en la razón sus posiciones; la ciencia se expone, no se impone y hay que estar abierto a modificaciones de los constructos existentes a partir del descubrimiento de nuevas realidades.

Los seis puntos expuestos como desafíos son, al mismo tiempo, las oportunidades que tienen las universidades para desarrollarse en el presente siglo, enfatizando su autonomía, su complejidad, la agrupación de los docentes y alumnos -especialmente los académicos que son islas y no comunican sus descubrimientos a sus pares-, la exclusividad de la búsqueda del saber otorgando sólo ellas los grados académicos y reforzando la disputa como método permanente de aprendizaje, sin olvidar los medios de presión cuando sean necesarios.

\section{IV.- La universidad y la realidad chilena}


Por último, al centrarse más en la realidad chilena, a partir de ella, se harán algunas reflexiones de los últimos tiempos y del futuro:

1.- Universidad para Todos: Este es un antiguo slogan y deseo que ha emergido en diferentes latitudes hace algunas décadas atrás. En el caso de Chile ello se ha ido transformando en una necesidad y exigencia de la sociedad, puesto que la cobertura del sistema escolar en la educación básica (primaria en otros países) está en el 98\% y en la educación media (secundaria en otros países) está bordeando el 90\%; luego, al existir una cultura de que lo único importante para el futuro es poseer un título o grado universitario desprestigiando de paso a la educación profesional o técnica-, la gran mayoría pretenda ingresar a la Universidad, pero como en este país existe selección para el ingreso, no todos pueden hacerlo y se produce la consiguiente frustración. A mi entender, la universidad no es para todos y a ella deben acceder quienes posean las aptitudes y capacidades suficientes para dedicarse a la academia, a lo que es el origen de la universidad, es decir, profundizar y desarrollar el saber en sus diferentes grados. La universidad, en su esencia, no es para otorgar sólo títulos profesionales; ello fue sólo una exigencia de la época moderna ${ }^{11}$, distorsionando, en parte, la razón de ser de la universidad: búsqueda del saber a través de la investigación con el fin de desarrollar las artes, las ciencias, la filosofía y la teología; ello no es más que cumplir en la actualidad lo que los artesanos del espíritu postulaban en los orígenes fundacionales de la universidad.

Sobre este punto también ha habido errores de política en Chile. Como la educación superior (universidades, institutos profesionales y centros de formación técnica) son pagados, el estado entrega becas y créditos a los estudiantes de este nivel educacional; durante varios años se privilegió casi exclusivamente el otorgamiento de estos beneficios a los estudiantes universitarios, dejando de lado a los de institutos profesionales y centros de formación técnica; sólo hace unos tres años, se ha estado corrigiendo tal error, entregando también beneficios a los estudiantes de las dos últimas instituciones. Para entregar tales beneficios, se exige que las personas demuestren que pertenecen a los quintiles más bajos de la población y que las entidades en que estudiarán estén acreditadas.

Desde un punto de vista del desarrollo del país, se ha demostrado en reiteradas oportunidades que la pirámide educacional no es tal, pues por cada 4 universitarios hay un técnico y lo que el país necesita es 4 técnicos por cada universitario.

2.- La inclusión es otro aspecto que en el ámbito educacional se ha venido insistiendo en él en las últimas décadas. Chile ha tenido a lo largo de su historia republicana una especial preocupación por este aspecto. En efecto, los diferentes gobiernos han elaborado y aplicado políticas públicas con el objeto de que todos sus niños y jóvenes ingresen al sistema escolar. Aunque los efectos de ello se percibían en menor grado durante el siglo XIX, esta situación se fue revirtiendo durante el siglo pasado, logrando los efectos esperados en los últimos años. Especial importancia tienen los esfuerzos por elevar la cantidad de años que el niño y joven permanezcan en el sistema escolar (12 años recientemente $)^{12}$; las campañas de alfabetización en los diferentes períodos de la segunda mitad del siglo pasado, que han llevado a que la tasa actual esté en un 96\%; los programas especiales hacia las etnias mapuche y aymara de las últimas décadas y el programa de mejoramiento de la calidad y equidad de la educación desarrollado en los últimos veinte años, cuya finalidad es que los niños y jóvenes más vulnerables recibían también una educación de calidad para poder enfrentar debidamente el competitivo mundo laboral actual $^{13}$ 
Durante el siglo XX tuvieron especial importancia los planteamientos de Dewey, en cuanto a que la educación favorecía la democracia; ellos ayudaron en Chile a crear una idea generalizada: que la educación debía estar accesible para todos, es decir, reforzar la política de inclusión al sistema educativo ${ }^{14}$.

En relación a la inclusión en la educación universitaria, a través de las becas y los créditos, se ha hecho accesible a jóvenes de menores ingresos; sin embargo, no hay que olvidar lo señalado en el punto anterior: La universidad no es para todos.

3.- La acreditación es otro fenómeno a nivel de la educación universitaria que desde hace cerca de dos décadas ha estado presente. En Chile se promulgó el 23 de octubre del 2006 y se publicó en el Diario Oficial el 17 de noviembre del mismo año, la Ley $\mathrm{N}^{\circ} 20.129$ de "Aseguramiento de la Calidad de la Educación Superior"; a través de ella se creó, según el Art. 6, la Comisión Nacional de Acreditación -CNA-; en el Art. 1 se establecen las funciones básicas del Aseguramiento de la Calidad, las cuales debe desarrollar la Comisión: Acreditación institucional, acreditación de carreras y difusión de la información para conocimiento de la opinión pública. La acreditación se otorga entre 2 y 7 años, correspondiendo a la cantidad de los años entregados, la calidad institucional o de las carreras ofrecidas; aunque la ley señala que la acreditación es voluntaria (Art. 15) en el Art. 27 se señalan algunas carreras que deberán obligadamente acreditarse: Médico Cirujano, Profesor de Educación Básica, Profesor de Educación Media, Profesor de Educación Diferencial y Educador de Párvulos. Para acreditar las carreras existirán agencias acreditadoras (Art. 26) que pueden ser nacionales o extranjeras ${ }^{15}$.

Nadie ha discutido fundadamente el por qué de la acreditación. Al autor de esta exposición le parece que ello es así porque se han autorizado que existan una serie de instituciones como universidades sin tener los requisitos para hacerlo y menos el cumplir lo que es realmente una universidad, según su origen, es decir, una institución que investiga para entregar una buena docencia, que difunde lo descubierto y que desarrolla las artes, las ciencias, la filosofía y la teología, o sea, que es una universidad compleja. En el futuro de la educación universitaria se encuentra la esperanza de que tenga que desaparecer la acreditación porque todas las instituciones existentes cumplen con la razón de ser de la universidad.

4.- Intercambio estudiantil y de maestros: Este punto es muy relevante, especialmente desde que se comenzó a producir la globalización de la información hace un par de décadas. Siempre ha existido intercambio de académicos y estudiantes, pero en la realidad actual este aspecto debe ser cada vez más in crescendo; en Chile ha ido aumentando considerablemente, tanto en las universidades públicas como privadas, a partir de la Declaración de Bolonia de 1999, puesto que ella incentiva a los países latinoamericanos, en este caso a Chile, para que estudiantes chilenos puedan estudiar en Europa y se beneficien de lo que significa tener un título o grado en Europa: Tendrán los mismos beneficios de un europeo, es decir, un título reconocido y compatible con la estructura de los estudios superiores de 46 países europeos, pudiendo pasar de un ciclo a otro sin necesidad de trámites de homologación. De allí la importancia de que universidades chilenas firmen convenios de intercambio con universidades europeas; los principales convenios de las universidades del Consejo de Rectores ${ }^{16}$ se encuentran en www.cruch.cl; a su vez, cada universidad posee sus propios Convenios de intercambio ${ }^{17}$. Las 
universidades privadas, especialmente las acreditadas, también poseen Convenios de intercambio.

En el futuro debieran reforzarse el intercambio de académicos -para que a lo menos cada cinco años realice un semestre académico en otra universidad y de otro país- y el intercambio estudiantil: todo alumno universitario debiera realizar, a lo menos, un semestre académico en una universidad de un país diferente. En la Unión Europea se han dado pasos muy significativos al respecto en los últimos años; todo ello, según la Declaración de Bolonia $^{18}$, firmada por 29 Ministros de Educación Europeos -tanto de la Unión Europea como de otros países como Rusia y Turquía-, el 19 de junio de 1999. Esta Declaración dio inicio a un verdadero proceso de convergencia que perseguía facilitar el intercambio de titulados o graduados y adaptar el contenido y duración de los estudios universitarios a las demandas sociales y a la realidad actual del conocimiento. Esta Declaración, a su vez, condujo a la creación del Espacio Europeo de Educación Superior, al cual se incorporaron diferentes países y servirá de marco de referencia a las reformas educativas que muchos países han iniciado a comienzos de este siglo. En Chile ha significado que algunas universidades ya han acortado la duración de los pre-grados y de los post-grados; más aún, al cuarto año entregan la licenciatura y al quinto año, el magíster.

\section{V.- A modo de conclusión}

Chile a nivel particular y Latinoamérica a nivel general, tienen grandes desafíos y también oportunidades en el contexto de lo aquí señalado: Constituir sus universidades de acuerdo a los principios fundacionales de las mismas en la Edad Media; ello permitiría mayor autonomía; universidades más complejas; maestros y estudiantes más unidos y colaborativos, pues el saber es patrimonio de todos; la entrega exclusiva de todos los grados académicos; mayor debate o discusión en el ejercicio de la investigación y la docencia y ojala sin necesidad de recurrir a la huelga. De esta forma, a su vez, se podrían enfrentar mejor los problemas aquí esbozados: Universidad para todos, la inclusión, la acreditación y el intercambio de maestros y estudiantes.

Santiago de Chile, enero de 2011. 


\section{Anexo $\mathrm{N}^{\circ}$ 1: Listado de Universidades Acreditadas de Chile}

- Pontificia Universidad Católica de Chile

- Pontificia Universidad Católica de Valparaíso

- Universidad Academia de Humanismo Cristiano

- Universidad Adolfo Ibáñez

- Universidad Adventista de Chile

- Universidad Alberto Hurtado

- Universidad Austral de Chile

- Universidad Bernardo O Higgins

- Universidad Católica Cardenal Raúl Silva Henríquez

- Universidad Católica de La Santísima Concepción

- Universidad Católica del Maule

- Universidad Católica de Temuco

- Universidad Central de Chile

- Universidad de Antofagasta

- Universidad de Arte y Ciencias Sociales, ARCIS

- Universidad de Chile

- Universidad de La Frontera

- Universidad de Las Américas

- Universidad de La Serena

- Universidad del Bío-Bío

- Universidad del Desarrollo 
- Universidad de Los Andes

- Universidad de Los Lagos

- Universidad del Pacífico

- Universidad de Magallanes

- Universidad de Playa Ancha de Ciencias de la Educación

- Universidad de Santiago de Chile

- Universidad de Talca

- Universidad de Valparaíso

- Universidad de Viña del Mar

- Universidad Diego Portales

- Universidad Gabriela Mistral

- Universidad Iberoamericana de Ciencias y Tecnología UNICIT

- Universidad Internacional SEK

- Universidad Mayor

- Universidad Metropolitana de Ciencias de la Educación UMCE

- Universidad Nacional Andrés Bello

- Universidad San Sebastián

- Universidad Santo Tomás

- Universidad Técnica Federico Santa María

Recebido em: 03/03/11

Aprovado em: 30/32011

Anexo $\mathbf{N}^{\circ}$ 2: Listado de Universidades en proceso de acreditación de Chile

- Universidad de Tarapacá

- Universidad Finis Terrae

- Universidad de Artes, Ciencias y Comunicación, UNIACC

- Universidad Arturo Prat

- Universidad Tecnológica de Chile - INACAP

- Universidad Autónoma de Chile

- Universidad de Atacama

- Universidad Católica del Norte

- Universidad de Concepción

\section{Anexo $N^{\circ}$ 3: Listado de Universidades no acreditadas de Chile}

- Universidad Bolivariana

- Universidad Chileno - Británica de Cultura

- Universidad de Aconcagua

- Universidad de Ciencias de la Informática, UCINF

- Universidad del Mar

- Universidad Gabriela Mistral

- Universidad Internacional SEK

- Universidad Los Leones

- Universidad Miguel de Cervantes

- Universidad Pedro de Valdivia 
- Universidad Regional San Marcos

- Universidad Tecnológica Metropolitana UTEM

Notas:

$1 \quad$ Participación en el Foro Panel “Oportunidades y desafíos de la Educación Universitaria en Argentina y Latinoamérica en el Siglo XXI" realizado en la Universidad Nacional de Cuyo en Mendoza en el contexto del Doctorado en Educación, 25 de enero de 2011.

2 Académico de la Universidad de Santiago de Chile.

3 Lo desarrollado en esta exposición tiene como trasfondo lo expuesto por el autor de este trabajo en Educación Superior en Chile y su Internacionalización en el Panel "Estrategias de Internacionalización de la Educación Superior Universitaria" organizado por el Programa de Doctorado de la Universidad Nacional de Cuyo, Mendoza, el 27 de enero de 2010 y publicado: Caiceo, Jaime (2010). "Educación Superior en Chile y su Internacionalización" en Revista HISTEDBR On-line, № 38, junhio - ISSN: 1676-2584, Universidade Estadual de Campinas, Brasil, pp. 12/23: http://www.histedbr.fae.unicamp.br/revista/edicoes/38/index.html

4 http://www.monografias.com/trabajos14/universidad-orig/universidad-orig.shtml.

5 http://www.portalplanetasedna.com.ar/universidad_medieval.htm.

6 Cfr. Caiceo, Jaime (2010) Op. cit., p. 13.

7 En Chile se usó durante los períodos de reforma universitaria en la década del 60 del siglo pasado, incluso antes que los movimientos de París de 1968. En efecto, en 1967 se realizaron las tomas en dos de las Universidades Católicas existentes a la fecha (la de Valparaíso y la de Santiago) y al año siguiente en la Universidad de Chile, lo cual permitió la Reforma de esas universidades.

licenciado es el que se otorga al alumno de una universidad que ha aprobado un programa de estudios que comprenda todos los aspectos esenciales de un área del conocimiento o de una disciplina determinada. El grado de Magíster es el que se otorga al alumno de una Universidad que ha aprobado un programa de estudios de profundización en una o más de las disciplinas de que se trate. Para optar al grado de Magíster se requiere tener grado de Licenciado o un título profesional cuyo nivel y contenido de estudios sean equivalentes a los necesarios para obtener el grado de Licenciado. El grado de Doctor es el máximo que puede otorgar una Universidad. Se confiere al alumno que ha obtenido un grado de Licenciado o Magíster en la respectiva disciplina y que ha aprobado un programa superior de estudios y de investigación, y acredita que quien lo posee tiene capacidad y conocimientos necesarios para efectuar investigaciones originales. En todo caso, además de la aprobación de cursos u otras actividades similares, un programa de Doctorado deberá contemplar necesariamente la elaboración, defensa y aprobación de una tesis, consistente en una investigación original, desarrollada en forma autónoma y que signifique una contribución a la disciplina de que se trate".

$9 \quad$ DFL N ${ }^{\circ} 5$ del 6 de febrero de 1981, publicado en el Diario Oficial el 16 de febrero de 1981, en su Art. 2 señala: "Corresponde a estos organismos otorgar toda clase de títulos profesionales con excepción de aquellos respecto de los cuales la ley requiera haber obtenido previamente el grado de Licenciado en una disciplina determinada. Podrán, además, otorgar títulos técnicos dentro del área o ámbito de las profesiones respecto de las cuales otorguen títulos profesionales".

$10 \quad$ DFL N ${ }^{\circ} 24$ del 7 de abril de 1981, publicado en el Diario Oficial el 16 de abril de 1981, en su Art 2 se indica: "Los organismos a que se refiere el artículo anterior (se refiere a los Centros de Formación Técnica), podrán otorgar el título de técnico”.

11 El positivismo modernista en su afán de reducir la realidad a los hechos y a los experimentos, produjo un nuevo materialismo, el cual produjo una degradación del espíritu a la razón, en una primera instancia, y, en una segunda, desde la razón a la inteligencia, y con ello se llegó a la habilidad, 
transformando de esta forma la esencia de la universidad: Del cultivo del espíritu a la mera habilidad, es decir, a la entrega de títulos profesionales. Cfr. Caiceo, Jaime (1988) "La Pedagogía Universitaria en el Marco de la Pontificia Universidad Católica de Chile: Visión desde la Perspectiva de la Filosofía de la Educación" en Aula 2000, Volumen 5, № 5, Temuco: Pontificia Universidad Católica de Chile-Temuco, pp. $5 / 18$.

12 Esta situación se inició en Chile con la dictación de la Ley de Instrucción Primaria Obligatoria en 1920 (Ley N 3654, promulgada en el Diario Oficial el 20 de agosto); en la Reforma Educacional de 1927, inspirada en los principios pedagógicos de Dewey, se eleva la educación primaria de 4 a 6 años de duración; situación que se eleva a 8 en la Reforma Educacional de 1965, transformando la antigua educación primaria en educación básica; recientemente, en el 2003, se elevó la obligatoriedad de la educación a 12 años -los 8 años de la educación básica y los 4 años de la educación media (antigua secundaria)-.

13 Cfr. Caiceo, Jaime (2.005) "Procesos de Inclusión y Exclusión en el Sistema Educativo Chileno durante los Siglos XIX y XX" en Perspectivas Educacionales, No 5, Santiago de Chile: Universidad Metropolitana de Ciencias de la Educación, pp. 75/82.

14 Cfr. Caiceo, Jaime (2.005) "Algunos Antecedentes sobre la Presencia de la Escuela Nueva en Chile durante el Siglo XX" en Anuario de Historia de la Educación, No 6, Buenos Aires: Sociedad Argentina de Historia de la Educación, Prometeo Libros, pp. 31/49.

15 En Anexo $\mathrm{N}^{\circ} 1$ se indican las 40 universidades acreditadas en Chile de un total de 61 existentes a diciembre de 2010 (65.5\%); en Anexo $\mathrm{N}^{\circ} 2$ se encuentran las 9 universidades en proceso de acreditación (14.75\%) y en Anexo $\mathrm{N}^{\circ} 3$ se señalan las 12 universidades que no se han acreditado (19.75\%). La fuente es la CNA-Chile en su página web: http://www.cnachile.cl/acreditacion/AC_INS_UNIVER.html

16 Para precisar lo que es el Consejo de Rectores, cfr. Caiceo, Jaime (2010) Op. cit., pp. 13/14.

17 Cfr. www.mineduc/educacionsuperior.cl

18 ¿Será casualidad que el acuerdo se tomó en el lugar de inicio de la primera universidad medieval o se hizo ex profeso?

Recebido em 03/03/2011

Aprovado em 30/03/2011 